\title{
Analysis of Biomedical Images based on Automated Methods of Image Registration
}

\author{
João Manuel R. S. Tavares \\ Instituto de Engenharia Mecânica e Gestão Industrial, Departamento de Engenharia Mecânica, \\ Faculdade de Engenharia, Universidade do Porto, Portugal \\ tavares@fe.up.pt, www.fe.up.pt/ tavares
}

\begin{abstract}
This paper gives a review of the methods that we have developed over the last few years to register 2D/3D images and image sequences. Initially, we will introduce a feature-based method, which will be followed up by a summary of the intensity-based methods. Then we will present some applications of our methods using biomedical images with more automated image analysis tasks. The main applications are related to plantar pressure images and SPECT brain images, including both static images and image sequences.
\end{abstract}

Keywords. Image Analysis, Image Segmentation, Image Matching, Image Fusion, Medical Imaging.

\section{Introduction}

Image registration, i.e., the process of transforming an image so that features contained in it are properly adjusted to the homologous features contained in a second image, is a paramount research topic. Computational methods of image registration are frequently applied to static bi-dimensional (2D) and tridimensional (3D) images, as well as to 2D and 3D image sequences. For example, in Biomedical Imaging, these methods have been assuming an essential role in supporting enhanced image-based analysis, by addressing the fusion of information acquired by different imaging techniques, from different directions or even acquired at different times. These computational methods are also used for more effective segmentation of bio structures, the building of more realistic models, and the extraction of more reliable information, among other complex tasks. Within image registration, there is image matching and image interpolation. Image matching establishes correspondences between the related features in two related images, and image interpolation is carried out as the transformations are applied to images in the discrete domain [1].

Here, we will introduce the computational methodologies for registering static images and image sequences that we have developed, including matching features presented in the input images. Furthermore, we present and discuss practical cases involving static images, image sequences and images acquired by different imaging techniques. Finally, the conclusions and future perspectives are pointed out. 


\section{$2 \quad$ Methods}

\subsection{Feature-based}

The feature-based method developed to register two static images starts by segmenting the features in each input image to be used in the registration process, in particular points, contours and surfaces. Afterwards, each feature segmented in one image is matched with the correspondent feature in the second input image and then the geometric transformation involved is computed and applied to one of the input images [26], Fig. 1.

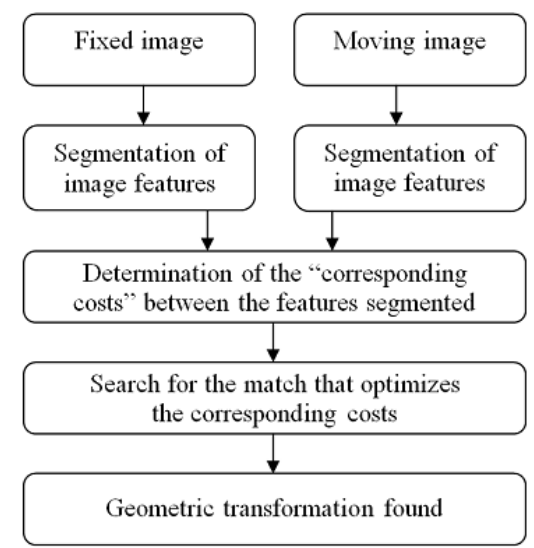

Fig. 1. Image registration method based on feature matching [2-6].

To find the best match among the segmented features, we have developed two approaches: 1) One is based on the physical modelling of the two sets of features, using the Finite Element Method, and on the modal space of each model built to search for the cost of each possible match based on the associated modal displacement. Then, the best match is found by applying an optimization algorithm that finds the lowest global matching cost. The two models are not necessary built using the same number of features, and so strategies to match the "extra" features have been developed. One such strategy is to add "non-realistic" features to the model with fewer features which can then be matched with the "real" more similar features. Strategies to attain matching of type "one-to-many" and vice-versa have also been developed, complementary to the traditional matching "one-to-one" [2-4]. 2) The other approach is based on the geometrical modelling of the two sets of features, using curvature or/and distance measures, and then by establishing the best matches by comparing the displacements of the features in the modal space, as in the physical modelling based matching approach, or by finding the most similar geometrical characteristics, and by applying an optimization algorithm in both cases [4-6].

After the best match between the two sets of features is established, the estimation of the involved global geometrical transformation is found by minimizing the least square differences between the matches established. Finally, the transformation 
found, which copes with the rotation, translation and scaling, is applied similarly to all pixels of one of the input images.

These feature-based methods are very fast and extremely robust to large deformations and to intensity variations. Another possible advantage of these methods is that the registration can be based on morphological characteristics of the structures represented in the input images, such as the contours of the heart left ventricle. However, it should be noted that with this method considerable image data may not be used in the registration process, which can be undesirable in some applications.

\subsection{Intensity-based}

Besides the registration method based on image features, we have developed two methods based on all image data. Hence, in these methods, the intensity of all image pixels (or voxels in 3D images) is taken into account in the registration process.

The first method developed using all image data [7] starts by transforming the input images from the Cartesian space into the Fourier space, and is known as the Fourier-based method. Then, the properties of the Fourier transform are taken into account to find the translation, rotation and scaling involved. Finally, the geometrical transform found is applied to one of the input images, Fig. 2. This method is fast and robust to large global transformations.

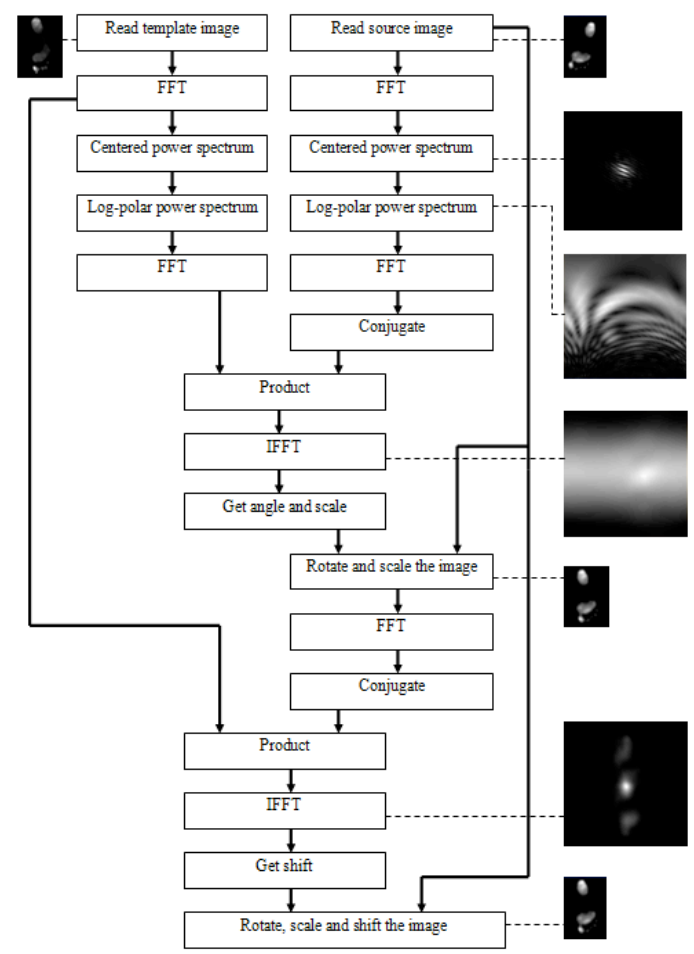

Fig. 2. Registration method based on Fourier transform [7]. 
The features- and Fourier-based methods can register two input images by applying the global transformation found to all pixels of one of the images; i.e. the input images are registered globally. However, in some cases, a local registration is desired, mainly for non-rigid registration applications, Fig. 3. To cope with these applications, which are very common, particularly in biomedicine, we have developed another method that changes one of the input images locally in order to maximize a similarity measure between the registered images, such as the mean squared error (MSE), by using an optimization algorithm [8].

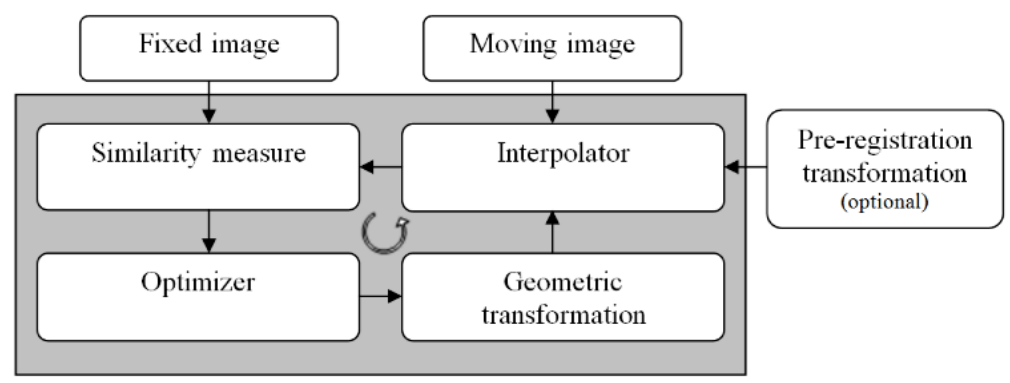

Fig. 3. Registration method based on local similarity optimization [8].

In comparison to the features- and Fourier-based methods, the local registration method is slower and also less robust to large deformations. Therefore, global based and local registration methods are often used combined: a feature- or Fourier-based method is used to perform an initial global registration, and then a "more local" method is used to improve the registration results locally. With this hybrid approach, we can obtain superior registration results that are extremely robust to large deformations. Also, a multi-resolution approach is commonly used to speedup the computational process.

Usually an interpolator is needed as the registration transformation found is applied to one of the input images that are defined in a discrete domain. We have used various different interpolators, such as those based on polynomials and on B-Splines. The interpolators based on B-Splines have shown excellent efficiency in various applications [9].

\subsection{Spatio-temporal registration}

Besides the previous methods that have been developed for static images, we have developed a method to register two image sequences in space and in time simultaneously [9-10]. This method starts by building a representative image for each input image sequence. Then, by using one of the previous global methods, i.e. the featuresor the Fourier-based method, the representative images are registered in space, and the transformation found is used to initially register the input sequences in terms of space combined with a linear temporal transformation to cope with the time registration. After this initial spatio-temporal registration, the registration is improved by 
optimizing a similarity measure both in space and time with an optimization algorithm, Fig. 4.

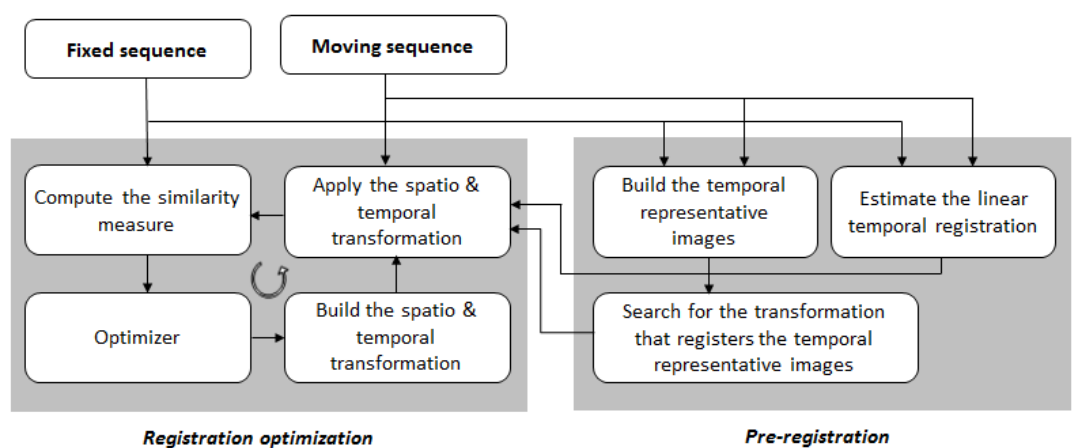

Fig. 4. Method developed to register two image sequences [9-10].

\section{$3 \quad$ Applications}

The methods that we have developed to register static images and image sequences have been applied to several experimental cases in order to facilitate tasks of complex image-based analysis. In this section, we present a few of these examples.

Fig. 5 shows an example of the registration of two 2D images and Fig. 6 two 3D medical images using the intensity-based method [1].

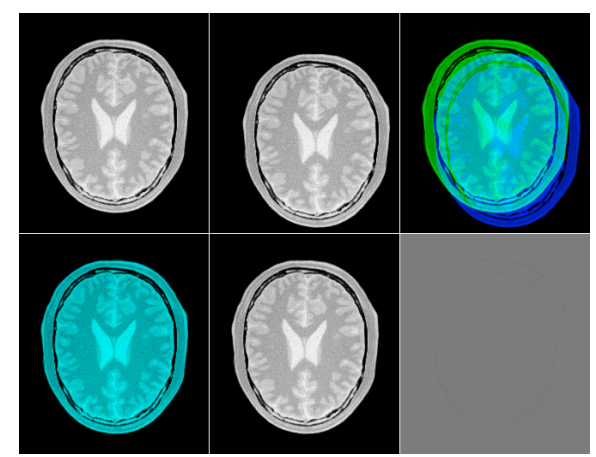

Fig. 5. Registration of two $2 \mathrm{D}$ images. Top: original gray-scale images and original image in pseudo-colour and overlapped before the registration. Bottom: images after the registration overlapped in pseudo-colour and in gray-scale, and the differences found between the two registered images.

One of the fields of application that deserves our particular attention is the analysis of plantar pressure images that are especially important when studying the diabetic foot, when designing better shoes or for plantar orthosis [7, 8, 11-13]. Fig. 7 shows clinically relevant pressure areas. These areas are identified by applying a registration method that facilitates the segmentation process, thus normalizing the foot under 
analysis in terms of size, position and orientation and then comparing the normalized foot against a template image. The same approach is used to classify the input image of a left or a right foot.
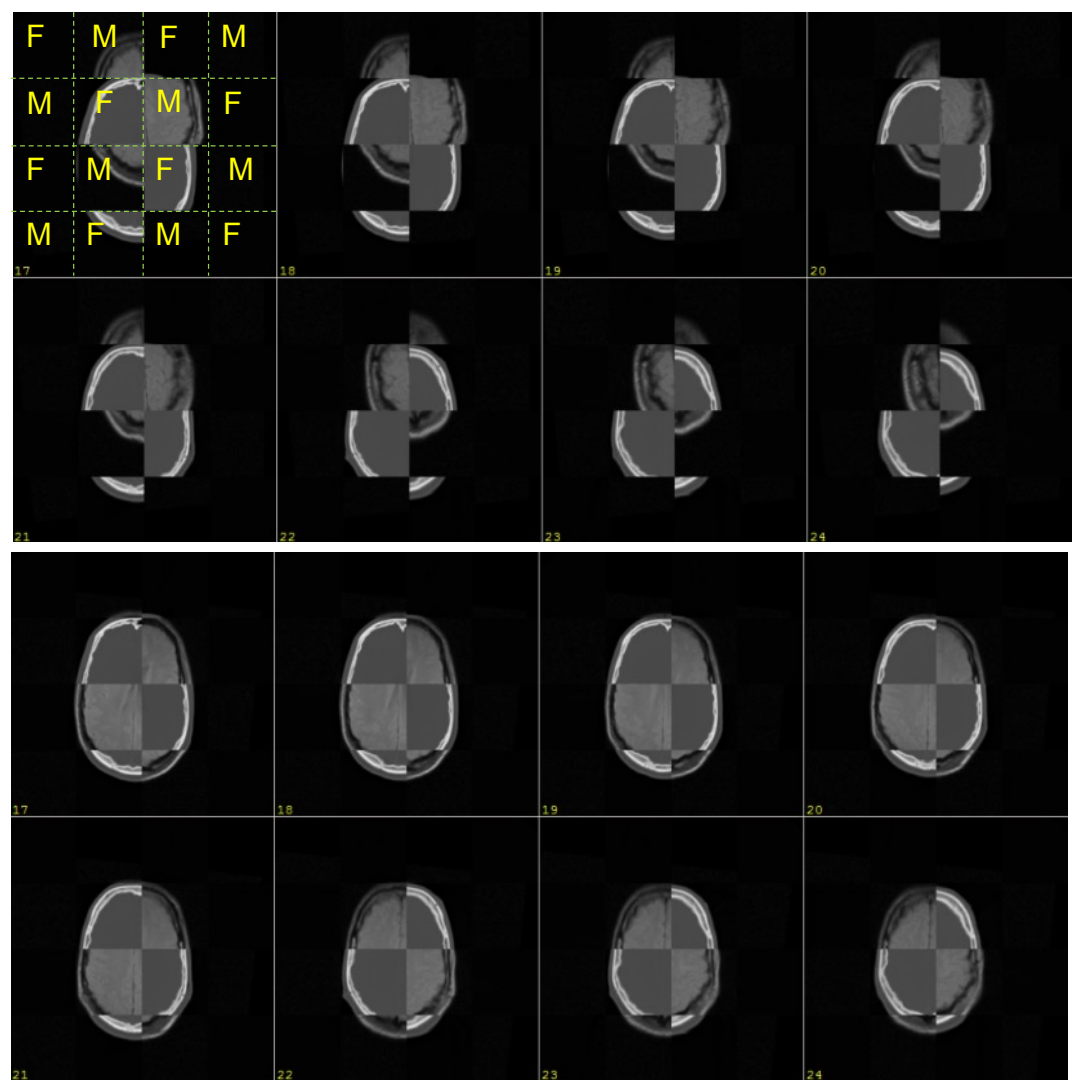

Fig. 6. Registration of two 3D images. Top: corresponding slices before the registration process - each slice shown was built using patches ( $F$ and $M$ ) that were cut from each input image dataset alternatively and, as can be seen, the structures are wrongly matched before the registration. Bottom: corresponding slices after the registration, where the structures are now well matched [1].

We have also used our methods to register plantar pressure image sequences in order to facilitate biomechanical studies by overcoming speed variations and distinct starting and ending instants of different image sequences, Fig. 8. Our results have shown that the registration methods are efficient to build representative plantar images and image sequences for subjects and populations. Hence, the computational and automated registration of the input images can lead to setting up "plantar pressure signatures" for subjects and populations.

Another important area that has gained our attention is the registration of nuclear medicine brain scan images, mainly SPECT brain images in order to assist in the di- 
agnosis of Parkinson's disease [14]. Briefly, the complex segmentation process of the images to be analysed is accomplished by registering the input images with a known template. After the segmentation of the ROIs in the input image, the potential of the ganglia basal is computed and the 3D shape of the ganglia basal is reconstructed, Fig. 9. Several measures are then calculated and used in a computational classifier in order to assist the clinical diagnosis.

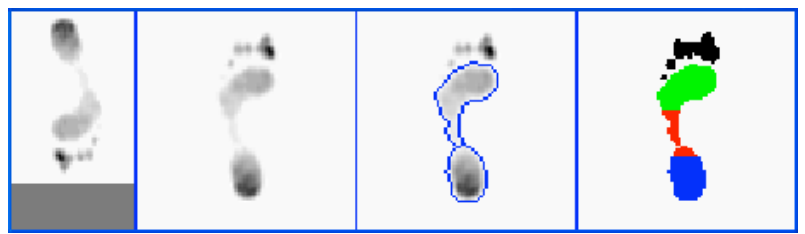

Fig. 7. Analysis of a plantar pressure image, left to right: original image, image after size, position and orientation normalization, segmentation of the main plantar region and identification of the important plantar regions [12].

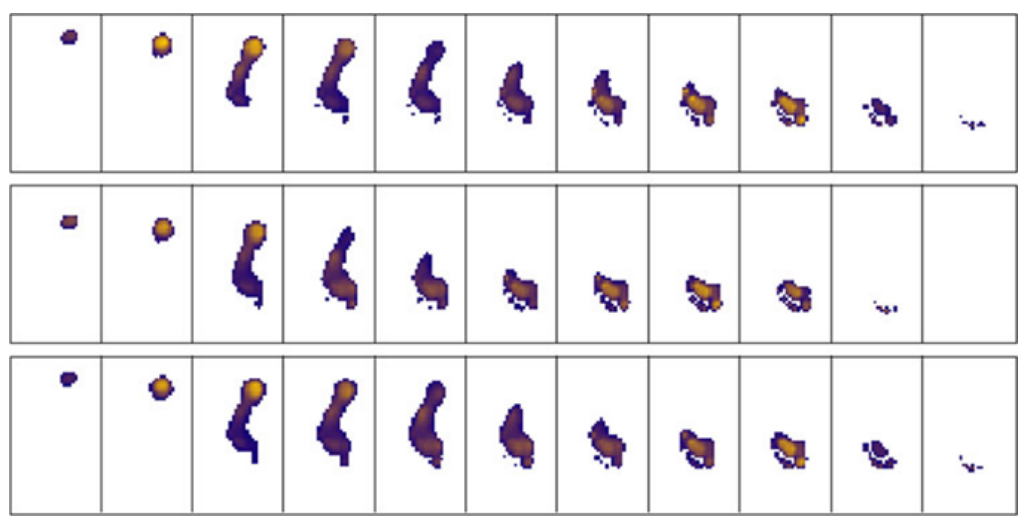

Fig. 8. Registration of two plantar pressure images. $1^{\text {st }}$ and $2^{\text {nd }}$ rows: original image sequences. $3^{\text {rd }}$ row: second image sequence after the registration process $[9,10]$.

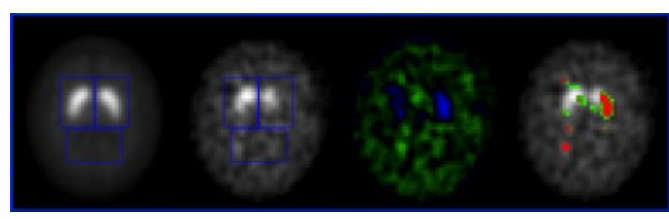

a)

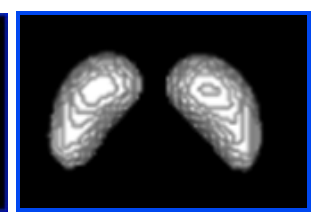

c)

Fig. 9. Identification of the ROIs (rectangles in blue) in SPECT brain images (a), results of a statistical comparison relative to a population representative template (b), and the $3 \mathrm{D}$ reconstruction of the ganglia basal based on image registration from SPECT brain images (c) [14].

Recently, we have also used image registration in order to obtain enhanced 3D reconstruction of structures in medical images acquired from different directions or differ- 
ent imaging modalities [15]. Fig. 10 shows the 3D shape of the bladder which was reconstructed using information from the sagittal and axial planes.

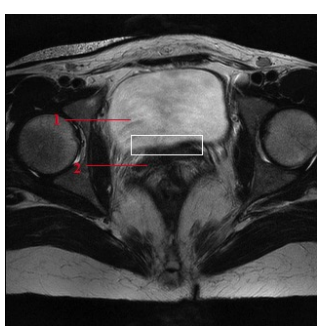

a)

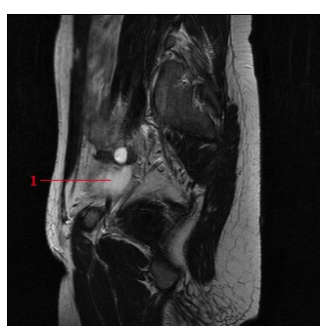

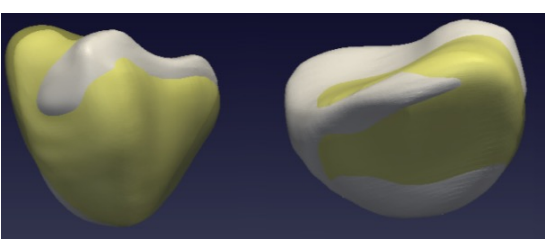

b)

Fig. 10. Magnetic resonance images of the axial and sagittal planes (a) and two views of the 3D reconstruction of the bladder based on the fusion of the data from those planes (b) [15].

\section{Conclusions}

The methods and applications, briefly reviewed here, were developed to register 2D/3D images and image sequences and they have been applied in several applications successfully.

As the main conclusions, we can state that the feature-based methods are faster and more robust to large deformations than the intensity-based methods. However, superior registration results can be obtained if a local minimization process is carried out. Also, the feature-based methods can be more morphologically oriented, which is an advantage in cases with images acquired using different imaging modalities, and when the information gathered from the intensities of the images is not as reliable as their registration. This is particularly important with biomedical images as the shape of the structures involved has primordial importance.

Several complex image analyses can be facilitated by using various registration methods, including the segmentation of low resolution images, the building of enhanced geometrical models by fusion of the information attained from different directions or/and imaging techniques, the recognition of partially occluded structures, the building of representative images for subjects and populations and the performing of statistical studies and comparisons.

In terms of future applications, we are presently working on cardiac nuclear medicine images using an analogous approach to the one used for the analysis of the nuclear medicine brain scan images. Also, a similar approach is now under developing to facilitate the segmentation of ear images, which is a complex task because of the reduced sizes of the structures involved in such images acquired using traditional CT systems. In spite of such difficulties, very promising results have already been achieved, Fig. 11. 


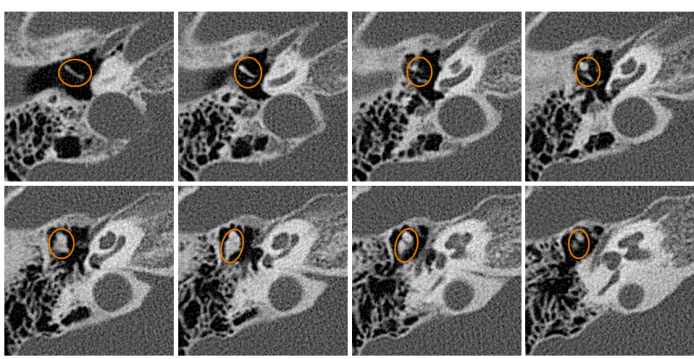

a)

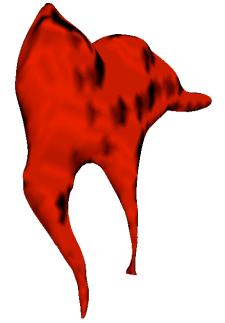

b)

Fig. 11. 3D visualization of the incus and malleus surface built (b) from the automated segmentation based on image registration of 3D CT images (a) (the ossicles are inside the ellipses drawn in each slice) [16].

\section{Acknowledgment}

The works summarized and reported here have been partially developed in the scope of the project "A novel framework for Supervised Mobile Assessment and Risk Triage of Skin lesions via Non-invasive Screening", with reference PTDC/BBBBMD/3088/2012, financially supported by Fundação para a Ciência e a Tecnologia (FCT) in Portugal.

\section{References}

1. Oliveira, F.P.M., Tavares, J.M.R.S.: Medical image registration: a review. Comput Method Biomec 17, 73-93 (2014).

2. Tavares, J.M.R.S., Barbosa, J., Padilha, A.J.: Matching image objects in dynamic pedobarography. In: RecPad 2000 - 11th Portuguese Conference on Pattern Recognition, Porto, Portugal (2000).

3. Tavares, J.M.R.S., Bastos, L.F.: Improvement of modal matching image objects in dynamic pedobarography using optimization techniques. In: Progress in Computer Vision and Image Analysis, Vol. 73, World Scientific, 339-368 (2010).

4. Bastos, L.F., Tavares, J.M.R.S.: Matching of objects nodal points improvement using optimization. Inverse Probl Sci En 14, 529-541 (2006).

5. Oliveira, F.P.M., Tavares, J.M.R.S.: Matching contours in images through the use of curvature, distance to centroid and global optimization with order-preserving constraint. CMES-Comp Model Eng 43, 91-110 (2009).

6. Oliveira, F.P.M., Tavares, J.M.R.S.: Algorithm of dynamic programming for optimization of the global matching between two contours defined by ordered points. CMES-Comp Model Eng 31, 1-11 (2008).

7. Oliveira, F.P.M., Pataky, T.C., Tavares, J.M.R.S.: Registration of pedobarographic image data in the frequency domain. Comput Method Biomec 13, 731-740 (2010).

8. Oliveira, F.P.M., Tavares, J.M.R.S.: Novel framework for registration of pedobarographic image data, Med Biol Eng Comput 49, 313-323 (2011). 
9. Oliveira, F.P.M., Sousa, A., Santos, R., Tavares, J.M.R.S.: Spatio-temporal alignment of pedobarographic image sequences. Med Biol Eng Comput 49, 843-850 (2011).

10. Oliveira, F.P.M., Tavares, J.M.R.S.: Enhanced Spatio-temporal alignment of plantar pressure image sequences using B-splines. Med Biol Eng Comput 51, 267-276 (2013).

11. Oliveira, F.P.M., Tavares, J.M.R.S.: Registration of plantar pressure images. Int J Numer Meth Bio 28, 589-603 (2012).

12. Oliveira, F.P.M., A. Sousa, R. Santos, Tavares, J.M.R.S.: Towards an efficient and robust foot classification from pedobarographic images. Comput Method Biomec 15, 1181-1188 (2012).

13. Oliveira, F.P.M., Pataky, T.C., Tavares, J.M.R.S.: Rapid pedobarographic image registration based on contour curvature and optimization. J Biomech 42, 2620-2623 (2009).

14. Oliveira, F.P.M., Faria, D.B., Costa, D.C., Tavares, J.M.R.S.: A robust computational solution for automated quantification of a specific binding ratio based on [123I]FP-CIT SPECT images. Q J Nucl Med Mol Im 58, 74-84 (2014).

15. Ma, Z., Jorge, R.N., Mascarenhas, T., Tavares, J.M.R.S.: A level set based algorithm to reconstruct the urinary bladder from multiple views. Med Eng Phys 35, 1819-1824 (2013).

16. Oliveira, F.P.M., Faria, D.B., Tavares, J.M.R.S.: Automated segmentation of the incus and malleus ossicles in conventional tri-dimensional computed tomography images. P I Mech Eng H 228, 810-818 (2014). 